\title{
Molecular characterization of human isolates of Strongyloides stercoralis and Rhabditis spp. based on mitochondrial cytochrome c oxidase subunit 1 (cox1)
}

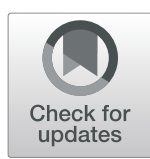

Mandana Fadaei Tehrani ${ }^{1}$, Meysam Sharifdini ${ }^{2}$, Farzaneh Zahabiun ${ }^{1}$, Robabeh Latifi ${ }^{1}$ and Eshrat Beigom Kia ${ }^{{ }^{*}}$

\begin{abstract}
Background: Due to the similarity of Strongyloides stercoralis with free-living nematodes of Rhabditis species they might be miss-diagnosed with each other in microscopical examination of stool samples. The aim of this study was molecular characterization and differentiation of human derived isolates of $\mathrm{S}$. stercoralis and Rhabditis species based on the mitochondrial gene of cytochrome c oxidase subunit 1 (cox1) amplification.

Methods: Using parasitological methods, ten isolates of S. stercoralis and three isolates of Rhabditis spp. were obtained from fresh stool samples of patients and the genomic DNA of the samples were extracted. PCR amplification of cox 1 gene was carried out for all the isolates and the products were sequenced.

Results: The phylogenetic analysis illustrated that $S$. stercoralis and Rhabditis spp. isolates were placed in two distinguishable separate clades. Inter-species genetic variation between isolates of $S$. stercoralis and Rhabditis spp. were ranged from 13.5 to $14.5 \%$.

Conclusions: Cox 1 gene was a suitable marker for discrimination of S. stercoralis from Rhabditis spp. retrieved from human in the current study. The availability of gene sequence information will be helpful in the future development and validation of discriminatory PCR-based assays of these nematodes.
\end{abstract}

Keywords: Strongyloides stercoralis, Rhabditis spp., PCR, cox1

\section{Background}

In genus Strongyloides, two species are known that infect human beings: Strongyloides fuelleborni that has local importance among human soil transmitted helminthes [1], and the soil-transmitted threadworm, Strongyloides stercoralis, which is one of the most neglected among neglected tropical diseases [2]. It is estimated that 30 to 100 million people are infected with S. stercoralis worldwide [1], mainly in tropical and subtropical areas. However, information on infection rates is missing in many countries [2]. In immunocompetent individuals, S. stercoralis commonly causes chronic, asymptomatic infection but a change in immune status can lead to an increase in

\footnotetext{
* Correspondence: keiaeshr@tums.ac.ir

'Department of Medical Parasitology and Mycology, School of Public Health,

Tehran University of Medical Sciences, Tehran, Iran

Full list of author information is available at the end of the article
}

parasite burden, hyperinfection syndrome, dissemination [3] and even death if not treated properly $[4,5]$. Corticosteroids have a particularly strong and specific association with the development of such infections; other immunosuppressive therapies and underlying conditions may also predispose to dissemination [3]. This imposes a serious threat to immunocompromised patients. Thus, an early laboratory diagnosis of this infection has special clinical importance.

Given the needs for the application of highly sensitive and specific methods for early diagnosis of strongyloidiasis, various parasitological, immunodiagnostic and molecular assays have been evaluated [6] and novel tests have been implemented [7]. However, a reliable gold standard for the diagnosis of this unique nematode is still lacking [6, 7]. Although molecular biology technique of polymerase chain reaction (PCR), that can detect

(c) The Author(s). 2019 Open Access This article is distributed under the terms of the Creative Commons Attribution 4.0 International License (http://creativecommons.org/licenses/by/4.0/), which permits unrestricted use, distribution, and 
DNA of the parasite, is highly specific, it should not yet be recommended for universal screening, nor as a standalone method for individual diagnosis of strongyloidiasis; and it has rather a role as a confirmatory test [7]. In the other words, in clinical parasitology, molecular diagnosis could be extremely useful when a specific parasite or group of parasites are to be studied, however they cannot yet replace microscopy as routine diagnostic tests [8]. In fact, routine microscopy is a catchall technique whereby the presence of any parasite in the clinical specimen can be detected [8].

There are many parasitological tests available for detection of S. stercoralis larvae in stool samples [6]. Agar plate culture (APC) is more efficient for detection of S. stercoralis in comparison with other parasitological tests including direct smear preparation and formalin-ether concentration [9]. Despite the efficiency of this culture method, it raises a possibility for the growth of other nematodes including hookworms [9], Trichostrongylus and free-living nematodes of the genus Rhabditis (Rhabditida) species [10]. In infectivity with the major soil transmitted nematodes of human, including Ascaris lumbricoides, Trichuris trichiura, and hookworms (Ancylostoma duodenale and Necator americanus) [1], the main diagnostic stage in stool sample is eggs [11]. While, Rhabditoid nematodes have been reported releasing larvae in human [12-15]. They are not among main helminthes of human [1], and there is no estimation on population infected in endemic areas of strongyloidiasis in the world. However, due to the similarity with S. stercoralis, in terms of the presence of larval stage in stool samples [12-15], their morphological differentiation is a challenge, requiring a well-trained microscopist.

Human infections with Rhabditis spp. nematodes have been reported as scattered sporadic reports from different parts of the world, for example from China [14], Korea [15], Japan [16], Brazil [13], Rhodesia [17], Germany [18] and Iran [12]. In the past, morphological criteria were used for discrimination of Rhabditis species from S. stercoralis $[12-17,19]$. However, given the increasing numbers of immunocompromised individuals throughout the world [20] and necessity for accurate and early detection of $S$. stercoralis, a more reliable method of differentiation is needed. Utilization of sensitive and specific diagnostic approach of molecular-based methods, associated with sequencing, will be helpful in this aspect. However, such utility is currently restricted due to lack of accurate gene sequence information of pathogenic species of Rhabditis. In this view, in order to pave the way for future development of an discriminative DNA based assay to be used in clinical setting, present study aimed to differentiate human derived isolates of S. stercoralis and Rhabditis species using molecular characterization of mitochondrial gene of cytochrome c oxidase subunit 1 (coxl) amplification.

\section{Methods}

\section{Sample collection}

This study was performed in 2016-2017. In order to obtain human isolates of $S$. stercoralis and Rhabditis spp., fresh stool samples were collected from patients who referred to the Diagnostic Laboratory of Strongyloidiasis in School of Public Health, Tehran University of Medical Sciences, and residents in endemic areas of strongyloidiasis in Iran [21] including Mazandaran, Guilan and Khuzestan Provinces.

\section{Parasitological methods}

Collected stool samples were examined by parasitological methods including direct smear preparation, formalin-ether concentration and nutrient agar plate culture (APC). For APC roughly $3 \mathrm{~g}$ of each stool sample was subjected to nutrient agar media [9]. Then, after $48-72 \mathrm{~h}$ incubation in room temperature at about $28{ }^{\circ} \mathrm{C}-30^{\circ} \mathrm{C}$, the plates were examined under a stereomicroscope for the presence of any larvae and free-living adults of S. stercoralis and Rhabditis species. In case of infectivity with Rhabditis spp., triple stool samples were taken to ensure true parasitic infection; and the patients were considered infected if all their triple stool samples had been found positive for Rhabditis species.

To prepare the infected samples for further molecular analysis, surface of nutrient agar plates were washed out with lukewarm phosphate buffer saline and after collection of larvae [10] they were preserved in $75 \%$ ethanol. Additionally, infected stool samples were also preserved in $75 \%$ ethanol after washing by sterile distilled water and sedimentation by centrifuge.

\section{DNA extraction}

Ethanol preserved cultivated and harvested larvae, and stool sediments containing larvae were washed three times with sterile distilled water by centrifugation at $5000 \times \mathrm{g}$ for $5 \mathrm{~min}$ to remove ethanol. Then, they were subjected to five cycles of freezing in liquid nitrogen and thawing in boiling water. Following this, approximately $300 \mathrm{mg}$ glass beads $(0.5 \mathrm{~mm}$ in diameter $)$ were added and shaken intensively for $5 \mathrm{~min}$. Subsequently, the genomic DNA was extracted by genomic DNA extraction kit (GeneAll Exgene, Seoul, Korea) according to the manufacturer's instructions and stored at $-20^{\circ} \mathrm{C}$ until the performance of PCR amplification.

\section{PCR amplifications and sequencing}

Polymerase chain reactions (PCR) based on the cox 1 gene were performed with primers cox F (5'TGG TTT GGG TAC TAG TTG-3') and cox R (5'-GAT GAG CTC AAA CTA CAC A-3') [22]. Every PCR amplification was carried out in a final reaction mixture containing $15 \mu \mathrm{L}$ of PCR mix including $1.25 \mathrm{U}$ Taq DNA 
polymerase, $200 \mu \mathrm{M}$ of dNTPs and $1.5 \mathrm{mM} \mathrm{MgCl} 22 \mathrm{x}$ red PCR Master Mix (Ampliqon, Odense, Denmark), 20 pmol of each primer and $2 \mu \mathrm{L}$ of each sample DNA. A negative control (distilled water) and a positive control (extracted DNA from S. stercoralis filariform larva) were applied in each run. The thermal PCR profile (Applied Biosystems 2720 Thermal Cycler, California, USA), included an initial denaturation step at $95^{\circ} \mathrm{C}$ for $6 \mathrm{~min}$, followed by 35 cycles of denaturation at $95^{\circ} \mathrm{C}$ for $45 \mathrm{~s}$, annealing at $55^{\circ} \mathrm{C}$ for $60 \mathrm{~s}$ and extension at $72^{\circ} \mathrm{C}$ for 60 s, followed by a final extension step at $72{ }^{\circ} \mathrm{C}$ for $6 \mathrm{~min}$.

The amplified products were run on a $1.5 \%$ agarose gel and visualized using a UV transilluminator.

These PCR products were submitted to Bioneer Company (South Korea) and were sequenced with Sanger method in both directions using the same PCR primers. All obtained sequences were edited and trimmed by Chromas software version 2.6.1 (South Brisbane, Australia).

Analysis of sequencing data was carried out using the National Center for Biotechnology Information BLAST programs and databases (http://www.ncbi.nlm.nih.gov/). Multiple sequence alignments were performed with Clustal W method using Bioedit software version 7.1 (http:// www.mbio.ncsu.edu/bioedit/bioedit.html) and compared with the sequences present in GenBank database.

\section{Phylogenetic analysis}

The phylogenetic tree was constructed using MaximumLikelihood algorithm and Tamura-3-Parameter option by Molecular Evolutionary Genetics Analysis software version 6.0 (MEGA). Bootstrap with 1000 replications was utilized for determining the topology reliability of the tree.

\section{Results}

In this study, overall ten isolates of S. stercoralis and three isolates of Rhabditis spp. were obtained from human stool samples. These isolates are listed in Table 1 to show the original province of each patient and the corresponded accession number submitted in GenBank.

After optimizing PCR conditions in terms of materials and thermal PCR protocol and the number of cycles, all S. stercoralis and Rhabditis spp. isolates were successfully demonstrated the amplification of about 509-bp target band for the coxl (Fig. 1). The tests were reliable when the negative control did not yield an amplification band and the positive control replicated the desired amplification band without presenting smears.

After trimming the sequences, the isolates were aligned and compared with the sequences available in GenBank. The sequence alignment indicated that there is high level of sequence difference between isolates of $S$. stercoralis and Rhabditis spp. (Fig. 2).

Inter-species and intra-species genetic variation between present isolates of S. stercoralis and Rhabditis spp.
Table 1 List of Rhabditis spp. (R1, R2, and R3) and Strongyloides stercoralis (S1 to S10) isolates with original province of patients and related accession numbers in GenBank based on amplification of cox 1 gene

\begin{tabular}{lll}
\hline Isolate & $\begin{array}{l}\text { Patient } \\
\text { original } \\
\text { province }\end{array}$ & $\begin{array}{l}\text { Accession } \\
\text { number } \\
\text { in GenBank }\end{array}$ \\
\hline R1 & Tehran & MG251327 \\
R2 & Mazandaran & MG251328 \\
R3 & Mazandaran & MG251329 \\
S1 & Guilan & MG251318 \\
S2 & Mazandaran & MG251319 \\
S3 & Mazandaran & MG251320 \\
S4 & Guilan & MG251321 \\
S5 & Khouzestan & MG251322 \\
S6 & Guilan & MG251323 \\
S7 & Khouzestan & MG251324 \\
S8 & Tehran & MG251326 \\
S9 & Mazandaran & MG251325 \\
S10 & Guilan & MG251317 \\
\hline
\end{tabular}

were calculated. The inter-species genetic variation between S. stercoralis and Rhabditis spp. were ranged from 13.5 to $14.5 \%$. Pairwise distance indicated the presence of intra-species genetic variation of $0-3.5 \%$ among the current isolates of S. stercoralis, and 0 to $5.2 \%$ of those with the sequences previously available in GenBank. Furthermore, multiple alignment represented that intraspecies similarity among Rhabditis spp. isolates was $100 \%$.

The phylogenetic tree was constructed based on cox 1 to evaluate the haplotypes recovered in this study along with retrieved sequences from other regions of the world

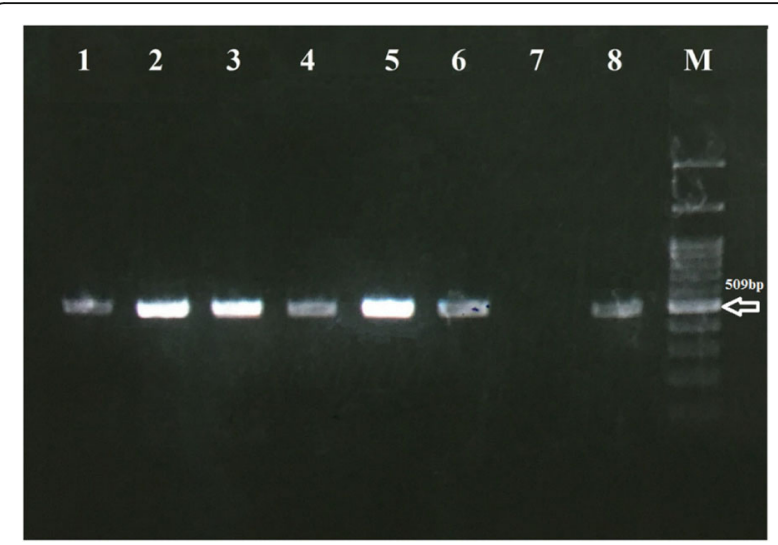

Fig. 1 Agarose-gel electrophoresis of polymerase chain reaction (PCR) products amplified with genomic DNA from Strongyloides stercoralis and Rhabditis spp. samples. Lanes 1, 2 and 3: S. stercoralis samples; Lanes 4, 5 and 6: Rhabditis spp. samples; Lane 7: Negative control; Lane 8: Positive control (S. stercoralis filariform larva); and Lane M: 100-bp DNA ladder 


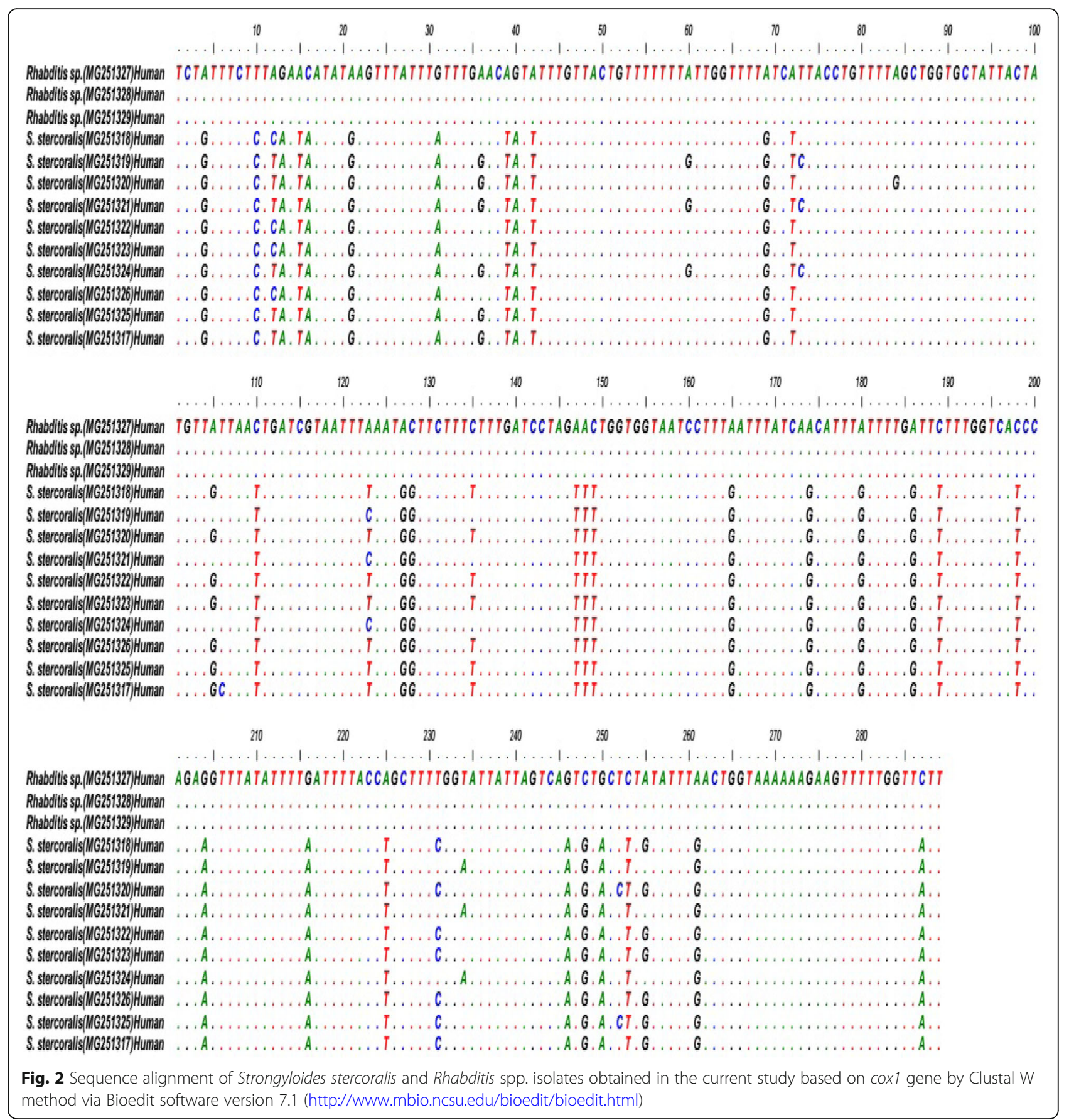

(Fig. 3). The phylogenetic analysis illustrated that S. stercoralis and Rhabditis spp. isolates were placed in two distinguishable separate clades. The tree indicated that the ten sequences of $S$. stercoralis isolates in the present study were consisted of five haplotypes, placing into two clusters. One cluster included four haplotypes whereas the other one consisted of merely one haplotype (Fig. 3). Intra-species genetic variation between the two clusters was calculated within 2.8 to $5.2 \%$.
The cox 1 sequences of all S. stercoralis and Rhabditis spp. were registered in GenBank database. The accession numbers of these sequences are shown in Table 1.

\section{Discussion}

Genus Rhabditis have been reported to infect human digestive system [12-15], urinary system [16, 17, 19], and even outer ear canal [18]; and excreting larvae in stool and urine. As the parasitological diagnosis of $S$. 


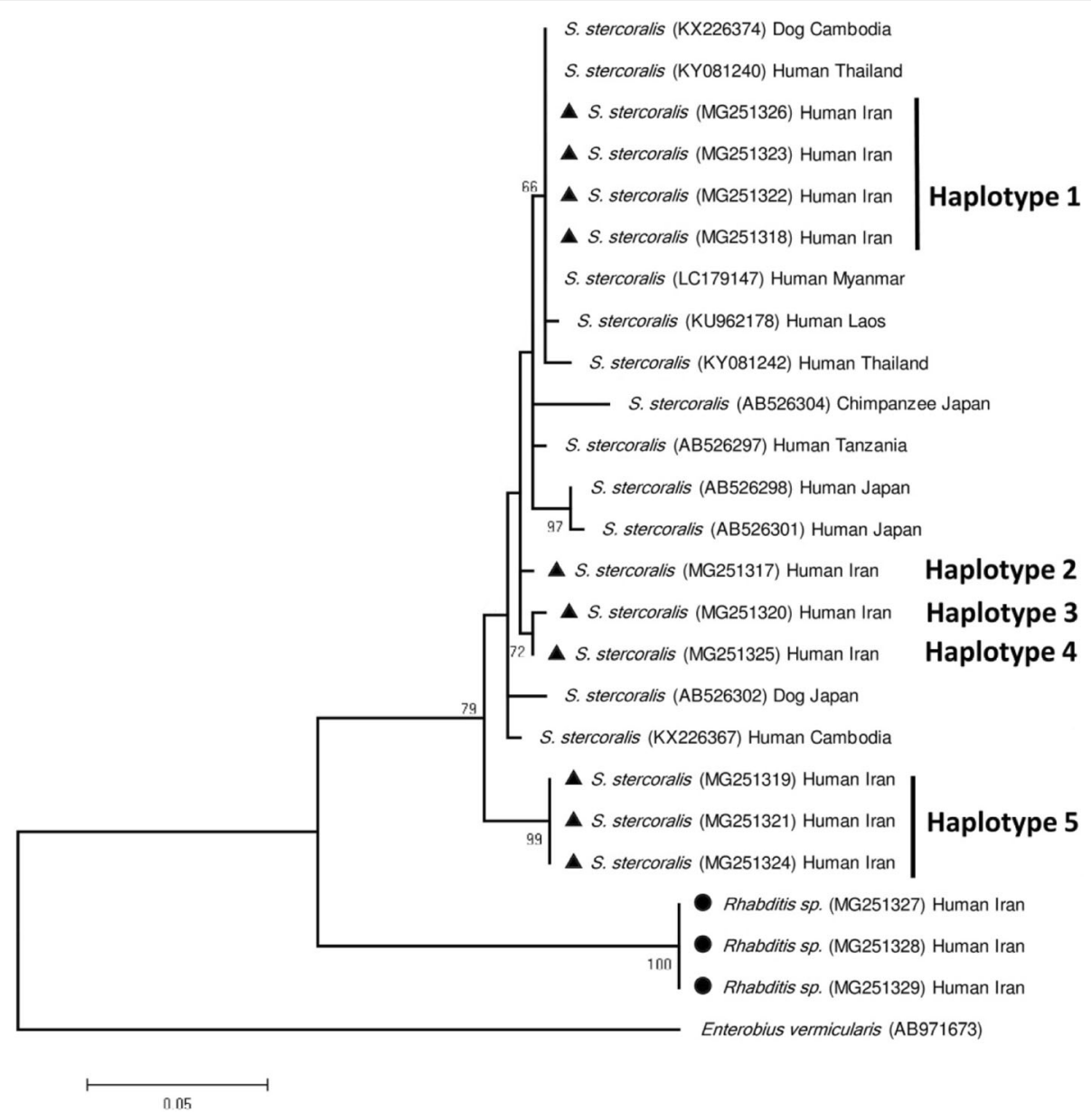

Fig. 3 Phylogenetic tree of Strongyloides stercoralis $(\boldsymbol{\Delta})$ and Rhabditis spp. ( $\bullet$ ) isolates obtained in this study and reference sequences retrieved from GenBank based on cox1 gene sequences and constructed tree using Tamura 3-parameter model by MEGA software version 6.0. Enterobius vermicularis was selected as out-group. Scale bar represented 0.05 changes per nucleotide

stercoralis is the detection of parasite larvae in stool exams and other biological materials such as sputum, duodenal aspirates, gastric biopsies, cervical smear or CSF liquid [6], therefore, this study pointing out to the challenge for discrimination between $S$. stercoralis and Rhabditis species. Due to the differences in the managements of infected patients with S. stercoralis [11], the exact differentiations of these two parasites is necessary.

The essential of early diagnosis of strongyloidiasis, especially in immunocompromised individuals, urged the efforts for development of molecular biology technique, mostly based on PCR, during recent years. Although, such techniques are highly sensitive and specific, they are recommended as confirmatory tests [7]. Besides, such molecular techniques have not been developed for detection of Rhabditis spp. recovered from human stool samples, and on the other hand, identification of such rare parasites using morphological criteria requires expert microscopists that may be lacking among new generation of laboratory staff [8]. Therefore, in order to attempt for prevention of misdiagnosis between these parasites, the present study was designed to characterize human retrieved isolates of S. stercoralis and Rhabditis spp. based on the amplification of $\operatorname{cox} 1$ gene. For this purpose, ten S. stercoralis and three Rhabditis spp. isolates were obtained from infected human stool samples and molecular approach was carried out by utilizing $\operatorname{cox} 1$ gene.

Although studies comparing quantitative polymerase chain reaction (qPCR) with microscopy methods for soil-transmitted helminthes are imperfect, they do show a significant increase in sensitivity and specificity of qPCR compared with labor-intensive traditional microscopic techniques [23]. The $\operatorname{cox} 1$ gene of mitochondrial DNA, which has been reported to mutate more rapidly than 18S rDNA, is useful sources of sequence data for study on different populations of Strongyloides species [24] and a useful target for molecular diagnosis of strongyloidiasis in human stool samples [22]. Presently, utilization of molecular-based methods in identification of Rhabditis species in human is very rare in clinical 
settings [18]. The sequence alignments of this study illustrated a considerable difference between nucleotide sequences of $S$. stercoralis and Rhabditis species. The inter-species genetic variation between $S$. stercoralis and Rhabditis spp. was 13.5 to $14.5 \%$. According to pairwise distance of the current study isolates, intra-species genetic variation within $S$. stercoralis nucleotide sequences was 0 to $3.5 \%$. Pairwise difference in $\operatorname{cox} 1$ gene among interbreeding strains of a nematode species was reported to be usually less than $6 \%$ and that between distinct species in a genus was more than 10\% [25]. Pairwise difference in nucleotides sequence of cox 1 gene among isolates of $S$. stercoralis from humans, apes and dogs was less than $4 \%$, regardless of the host and locality of the isolates [24]. The average pairwise distances of $\operatorname{cox} 1$ nucleotide sequences were 0.036 for $S$. stercoralis isolates from human and dog collected mainly from Myanmar [26].

According to the phylogenetic analysis of current study, S. stercoralis isolates were located in a distinct clade from Rhabditis species. Rhabditis spp. clade was included three isolates, all merely from our study, having $100 \%$ similarity with each other. There was not any available cox 1 gene sequence of Rhabditis species with human origin or other pathogenic isolates to be included in the constructed tree for comparison. The phylogenetic tree indicated that the ten sequences of S. stercoralis consisted of five haplotypes. The haplotype one, which included four isolates, showed 100\% homology with GenBank registered sequences of human isolates from Thailand (KY081240), Myanmar (LC179147), and dog isolate from Cambodia (KX226374). The haplotypes two, three and four, each including one isolate, have not been already recorded from other regions in the world. Moreover, haplotype five, locating solely in separate cluster and including three completely similar isolates, was distinctive from any other haplotypes in the tree. Despite the small number of $S$. stercoralis isolates studied presently, five different haplotypes were recovered which four of them were considered as new reports. Thus far, it seems S. stercoralis represents diverse haplotypes and needs to be speculated further using more isolates from different regions of the world in order to investigate its genetic variations.

Among our isolates of S. stercoralis, haplotype one indicated $100 \%$ homology with a dog isolate from Cambodia (KX226374), belonging to the population indistinguishable from the population of S. stercoralis isolated from humans in Cambodia [27]. In mentioned study, two genetically different populations of Strongyloides spp. were found in dogs, one of which that the majority of the worms belonged, appeared to be restricted to dogs; the other population was shared with humans [27]. In another study, using nuclear and mitochondrial markers, phylogenetic relationships among S. stercoralis isolates from several human and dog populations in multiple countries of East Asia were examined [26]. Accordingly, two distinct lineage of $S$. stercoralis were present, referring to as type A parasites isolated both from humans and dogs, and type B parasites founding exclusively in dogs and not adapted to infect humans. All these findings suggest the possibility of zoonotic potential of $S$. stercoralis and the possibility that human S. stercoralis originated from dogs. Thus, dogs might be considered as a reservoir for human S. stercoralis. Further studies with larger samples of human and dog isolates from different geographical areas is recommended for assessing this assumption particularly in region where people have close contact with dogs.

\section{Conclusion}

The present comparative molecular characterization of $S$. stercoralis and Rhabditis spp. on human-derived samples, based on $\operatorname{cox} 1$ gene, indicated that this gene is a suitable marker for discrimination of $S$. stercoralis from the species of Rhabditis obtained in the current study. Due to the lower occurrence of Rhabditis spp. in human, only three isolates were available for analysis. However, this is for the first time in the world that human-dervied Rhabditis spp. partial sequences of cox 1 gene is compared with human isolates of S. stercoralis and the sequences are now available in GeneBank database. In order to find whether these sequences are representative and conserved among other pathogenic species of Rhabditis spp. more human and even animal retrieved isolates from different geographical areas need to be analysed, with cox1 gene as well as other gene markers. The availability of accurate gene sequence information will be helpful in the future development and validation of discriminatory PCR-based assays, particularly ones with the low cost, rapid and simple method, for firstpass screening in research laboratories, clinical settings and epidemiological studies especially in regions with low resources.

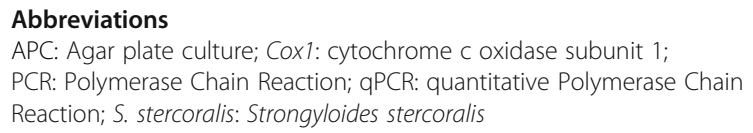

\section{Acknowledgments}

The authors wish to thank Mrs. Zohre Fakhrie Kashan for her kind assistance. The authors declare that there is no conflict of interests.

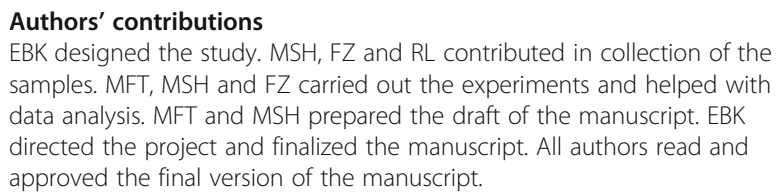

This study was part of the MSc Thesis of first author (M. Fadaei Tehrani) in the Department of Medical Parasitology and Mycology, School of Public Health, Tehran University of Medical Sciences. 
The study was financially supported by the Deputy of Research, Tehran University of Medical Sciences, through grant no. 96-04-27-35349. The funding body played no role in the design of the study, collection, analysis, interpretation of data or writing.

\section{Availability of data and materials}

The data supporting the conclusion of the article are included in the text of the article and its additional files. The accession numbers of sequences deposited in GenBank database are provided in the relevant table of the article.

\section{Ethics approval and consent to participate}

The study was approved by the Research and Ethics Committee of the Tehran University of Medical Sciences. The enrolled patients in the study were all among adult individuals whom were referred to the Diagnostic Laboratory of Strongyloidiasis in School of Public Health, Tehran University of Medical Sciences. Written informed consent was attained for each participant and confidentiality of the information was assured. The results of the diagnostics tests of the participants were delivered to them in confidential written format and all their diagnostics tests were free of charge.

\section{Consent for publication}

Not applicable.

\section{Competing interests}

The authors declare that they have no competing interests.

\section{Author details}

'Department of Medical Parasitology and Mycology, School of Public Health, Tehran University of Medical Sciences, Tehran, Iran. ${ }^{2}$ Department of Medical Parasitology and Mycology, School of Medicine, Guilan University of Medical Sciences, Rasht, Iran.

Received: 17 May 2019 Accepted: 25 August 2019

Published online: 05 September 2019

\section{References}

1. Bethony J, Brooker S, Albonico M, Geiger SM, Loukas A, Diemert D, et al. Soil-transmitted helminth infections: ascariasis, trichuriasis, and hookworm. Lancet. 2006:367(9521):1521-32.

2. Schar F, Trostdorf U, Giardina F, Khieu V, Muth S, Marti H, et al. Strongyloides stercoralis: global distribution and risk factors. PLoS Negl Trop Dis. 2013;7(7): e2288.2

3. Mejia R, Nutman TB. Screening, prevention, and treatment for hyperinfection syndrome and disseminated infections caused by Strongyloides stercoralis. Curr Opin Infect Dis. 2012;25(4):458-63.

4. Kia EB, Rahimi HR, Mirhendi H, Nilforoushan MR, Talebi A, Zahabiun F, et al. A case of fatal strongyloidiasis in a patient with chronic lymphocytic leukemia and molecular characterization of the isolate. Korean J Parasitol. 2008;46(4):261-3.

5. Meamar AR, Rezaian M, Mohraz M, Hadighi R, Kia EB. Strongyloides stercoralis hyper-infection syndrome in HIV+/AIDS patients in Iran. Parasitol Res. 2007; 101(3):663-5.

6. Requena-Mendez A, Chiodini P, Bisoffi Z, Buonfrate D, Gotuzzo E, Munoz J. The laboratory diagnosis and follow up of strongyloidiasis: a systematic review. PLoS Negl Trop Dis. 2013;7(1):e2002.

7. Buonfrate D, Requena-Mendez A, Angheben A, Cinquini M, Cruciani M, Fittipaldo A, et al. Accuracy of molecular biology techniques for the diagnosis of Strongyloides stercoralis infection-a systematic review and metaanalysis. PLoS Negl Trop Dis. 2018;12(2):e0006229.

8. Wong SS, Fung KS, Chau S, Poon RW, Wong SC, Yuen KY. Molecular diagnosis in clinical parasitology: when and why? Exp Biol Med. 2014; 239(11):1443-60.

9. Arakaki T, Iwanaga M, Kinjo F, Saito A, Asato R, Ikeshiro T. Efficacy of agarplate culture in detection of Strongyloides stercoralis infection. J Parasitol. 1990;76(3):425-8.

10. Kia EB, Mahmoudi M, Zahabiun F, Meamar AR. An evaluation on the efficacy of agar plate culture for detection of Strongyloides stercoralis. Iran J Parasitol. 2007;2(1):29-34
11. Krolewiecki AJ, Lammie P, Jacobson J, Gabrielli AF, Levecke B, Socias E, et al. A public health response against Strongyloides stercoralis: time to look at soil transmittedhelminthiasis in full. PLoS Negl Trop Dis. 2013;7(5):e2165.

12. Meamar AR, Kia EB, Zahabiun F, Jafari-Mehr A, Moghadam A, Sadjjadi SM. The occurrence of severe infections with Rhabditis axei in AIDS patients in Iran. J Helminthol. 2007:81(4):351-2.

13. Campos DM, Araujo JL, Vieira MC, Damasceno F, Barbosa AP. A case of parasitism by Rhabditis sp. in a child from Goiania, Goias, Brazil. Rev Soc Bras Med Trop. 2002;35(5):519-22.

14. Ye LP, Zhu CG, Zhang JN. Two cases of Rhaditis axei infections in human disgestive system. Chin J Schistosomiasis Cont. 2002;14:187.

15. Ahn YK, Chung PR, Lee KT. Rhabditis sp. infected cases in rural school children. Kisaengchunghak Chapchi. 1985;23(1):1-6.

16. Hara M, Nakazato H, Araki T, Iwata S. A case of urinary tract infection by a nematode larva (Rhabditis sp.). Kisechugaku Zasshi. 1974:23:48

17. Goldsmid JM. Rhabditis (Rhabditella) axei in the urine of an African in Rhodesia. J Helminthol. 1967;41(4):305-8.

18. Teschner M, Wurfel W, Sedlacek L, Suerbaum S, Tappe D, Hornef MW. Outer ear canal infection with Rhabditis $s p$. nematodes in a human. J Clin Microbiol. 2014:52(5):1793-5.

19. He YX, Jiang H. 3 human cases of urinary tract infection with Rhabditis. Ji Sheng Chong Xue Yu Ji Sheng Chong Bing Za Zhi. 1985:3(3):206-8.

20. Keiser PB, Nutman TB. Strongyloides stercoralis in the Immunocompromised Population. Clin Microbiol Rev. 2004:17(1):208-17.

21. Sharifdini M, Kia EB, Ashrafi K, Hosseini M, Mirhendi H, Mohebali M, et al. An analysis of clinical characteristics of Strongyloides stercoralis in 70 indigenous patients in Iran. Iran J Parasitol. 2014;9(2):155-62.

22. Sharifdini M, Mirhendi $H$, Ashrafi K, Hosseini M, Mohebali M, Khodadadi $H$, et al. Comparison of nested polymerase chain reaction and real-time polymerase chain reaction with parasitological methods for detection of Strongyloides stercoralis in human fecal samples. Am J Trop Med Hyg. 2015; 93(6):1285-91.

23. O'Connell EM, Nutman TB. Molecular diagnostics for soil-transmitted helminths. Am J Trop Med Hyg. 2016;95(3):508-13.

24. Hasegawa H, Sato H, Fujita S, Nguema PP, Nobusue K, Miyagi K, et al. Molecular identification of the causative agent of human strongyloidiasis acquired in Tanzania: dispersal and diversity of Strongyloides spp. and their hosts. Parasitol Int. 2010;59(3):407-13.

25. Blouin MS. Molecular prospecting for cryptic species of nematodes: mitochondrial DNA versus internal transcribed spacer. Int J Parasitol. 2002 32(5):527-31.

26. Nagayasu E, Aung M, Hortiwakul T, Hino A, Tanaka T, Higashiarakawa M, et al. A possible origin population of pathogenic intestinal nematodes, Strongyloides stercoralis, unveiled by molecular phylogeny. Sci Rep. 2017;7(1): 4844

27. Jaleta TG, Zhou S, Bemm FM, Schar F, Khieu V, Muth S, et al. Different but overlapping populations of Strongyloides stercoralis in dogs and humansdogs as a possible source for zoonotic strongyloidiasis. PLoS Negl Trop Dis. 2017;11(8):e0005752

\section{Publisher's Note}

Springer Nature remains neutral with regard to jurisdictional claims in published maps and institutional affiliations.

Ready to submit your research? Choose BMC and benefit from:

- fast, convenient online submission

- thorough peer review by experienced researchers in your field

- rapid publication on acceptance

- support for research data, including large and complex data types

- gold Open Access which fosters wider collaboration and increased citations

- maximum visibility for your research: over $100 \mathrm{M}$ website views per year

At BMC, research is always in progress.

Learn more biomedcentral.com/submissions 